\title{
THE EXPERIMENTAL INVESTIGATION OF THE BEAM TRANSPORTATION EFFICIENCY THROUGH THE AXIAL INJECTION SYSTEM OF THE U400 CYCLOTRON
}

\author{
I. Kalagin, I. Ivanenko, G. Gulbekian, \\ JINR, FLNR, Dubna, Moscow reg., Russia
}

\begin{abstract}
The axial injection system of the U400 cyclotron has been constructed and put into operation since the second half of 1996. With the aim of increasing the transportation and the acceleration coefficients of the injected beams the modernization of this system is done. The double buncher system (Sine and Line types) was put into operation in 1997-1998. The expected acceleration efficiency with the buncher systems is about $70 \%$. At present time, the efficiency for the possible beam intensities is in the region of $20 \%-60 \%$. The experimental results of the efficiency investigation of the beam transportation through the axial injection system are present. The results have shown that the main beam losses are at the spiral inflector entrance. The beam losses are actually depended on the beam intensity. The possible methods of decreasing the beam losses are proposed.
\end{abstract}

\section{INTRODUCTION}

The U-400 axial injection system has been operated since 1996 [1]. The system consists of the ECR4M- ion source, solenoidal focusing lens, the injection line at the $9.28 \mathrm{~m}$ length with two bending magnets that bend ion beam in horizontal and vertical planes at the angles of 120 and 90 degrees correspondingly and the spiral inflector. To provide the ion beam focusing at the spiral inflector entrance, three solenoids are used in the vertical part of the injection line. Because of presence of U-400 diffused magnetic field in the upper part of injection line at the field value of $80 \div 200$ Gauss, the line has magnetic screens to decrease the field value inside the line in about factor 10. Also, five stirring magnets are used to correct the ion beam deviation. For the U-400 cyclotron, the efficiency of capture into acceleration is no more than $5 \div 10 \%$ without buncher systems. To increase the efficiency, a sine buncher was installed in the vertical part of the injection line in 1997, and a linear one was installed in 1998. The linear buncher situated at $4.4 \mathrm{~m}$ and the sine one is placed at $1.25 \mathrm{~m}$ above the median plane. At present time, the bunchers operate simultaneously, and the efficiency value for ${ }_{48} \mathrm{Ca}^{5+}$ ions changes from about $38 \%$ to $23 \%$ with increasing the beam intensities in the injection line from $22 \mu \mathrm{A}$ to $75 \mu \mathrm{A}$. In the case of single operation of the sine or linear buncher, the efficiency is not more than $25 \%$ for the beam current of $30 \div 50 \mu \mathrm{A}$. From the other hand, numerical calculations of the buncher working regimes for the ions gave us the efficiency for single sine buncher is about $50 \%$ [1], for single linear buncher it is in the region of $80 \div 90 \%$ [2], and for combination of two bunchers the value is about $70 \%$. In the numerical calculations, the processes of ion beam focusing by solenoids and the space- charge effects at the drift distance between the bunchers and the inflector were not taken into account.

The experimental investigations of beam transportation efficiency have been undertaken to find the reasons of the efficiency decreasing.

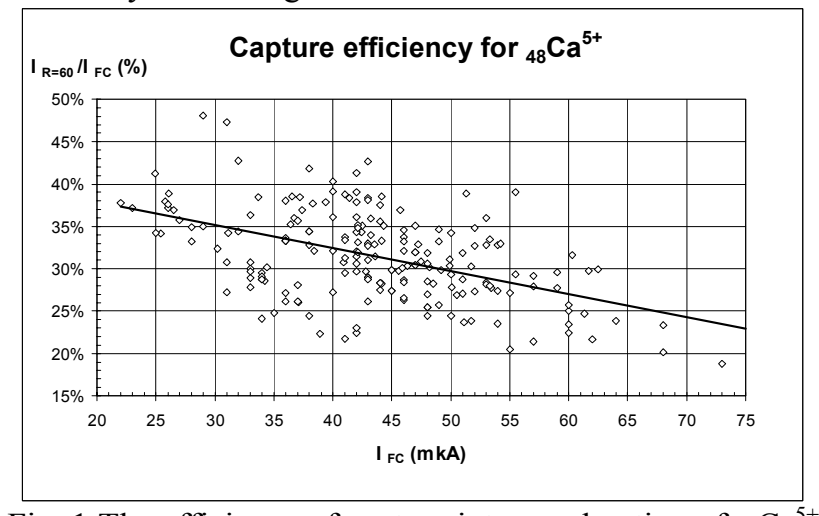

Fig. 1 The efficiency of capture into acceleration of ${ }_{48} \mathrm{Ca}^{5+}$ ions for the regime with two bunchers. The efficiency was calculated as ratio between the accelerated ion current at the radius of $R=0.6 \mathrm{~cm}$ inside the $U-400$ cyclotron $\left(I_{R=50}\right)$ and the ion current at injection line $\left(\mathrm{I}_{\mathrm{FC}}\right)$.

\section{MEASUREMENTS OF CAPTURE EFFICIENCY}

The capture into acceleration efficiency has been measured for rare isotope of ${ }_{48} \mathrm{Ca}\left({ }_{48} \mathrm{Ca}^{5+}\right.$ ions) and for ${ }_{40} \mathrm{Ar}^{4+}$ ions that have close to ${ }_{48} \mathrm{Ca}^{5+}$ ions mass to charge ratio. The efficiency was calculated as a ratio between the accelerated ion current at the radius of $\mathrm{R}=0.6 \mathrm{~m}$ inside the U-400 cyclotron (the vacuum losses of the ions are not more than $5 \%$ at the distance between the inflector and the radius) and the ion current at the injection line. To measure ion currents at the injection line a Faraday cup has been used, to measure currents inside the cyclotron a regular inner current probe of U-400 was used. The linear and sine bunchers operate simultaneously, the spans of voltage amplitudes on the bunchers are: $\mathrm{U}_{\mathrm{l}} \approx 180 \mathrm{~V}$ (linear buncher) and $\mathrm{U}_{\mathrm{s}} \approx 700 \mathrm{~V}$ (sine buncher). The results collected during the 3 months period of ${ }_{48} \mathrm{Ca}^{5+}$ acceleration are presented in the Fig. 1 The linear fitting gives us the rate of average efficiency in the region from $38 \%$ to $23 \%$ for the ion currents at injection line from 
$22 \mu \mathrm{A}$ to $75 \mu \mathrm{A}$. The efficiency dispersion depends on the ion source regimes ( $\mathrm{Ca}$ injected into ECR-plasma by evaporation from the oven), the vacuum conditions inside the cyclotron and the quality of the injection linecyclotron system adjusting.

To measure the efficiency more carefully, the ${ }_{40} \mathrm{Ar}^{4+}$ as a testing particle was used (the current of ${ }_{40} \mathrm{Ar}^{4+}$ ions from the ion source is stable enough). The results of the efficiency measurements for ${ }_{40} \mathrm{Ar}^{4+}$ ions are presented in the Fig. 2. The linear fitting gives us the rate of average efficiency is about $9 \%$ for the regime without bunchers at the ion currents in injection line from $2 \mu \mathrm{A}$ to $80 \mu \mathrm{A}$. The linear fitting for both linear and sine bunchers shows the average efficiency in the region from $58 \%$ to $23 \%$, for single linear buncher it changes from $48 \%$ to $10 \%$, for only one sine buncher it is between $29 \%$ and $15 \%$.

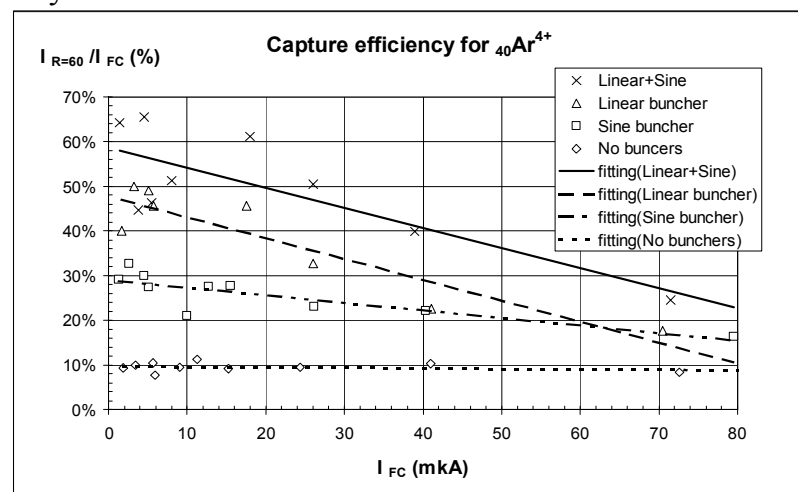

Fig. 2 The efficiency of capture into acceleration of ${ }_{40} \mathrm{Ar}^{4+}$ ions for the regimes without bunchers, with single linear buncher, with single sine buncher and with two bunchers.

The reasons of the efficiency decreasing in the regime with bunchers can be influence of space-charge effects, that become significant, because the DC ion current becomes pulsed one after the bunchers and the ion pulse current in a bunch in some times more the initial DC one. Also, the ion energy spread that can be up to $3 \%$ after the bunchers, in combination with longitudinal focussing magnetic field, can stimulate the effects of the beam cross-section increasing. As a result, the beam transverse emittance can be not matched to the acceptance of such element as the spiral inflector.

The influence of the construction of bunchers on the effects was studied in [1], [2] and optimisation of the buncher system was done.

The distribution of the beam current value along the injection line was measured for ${ }_{40} \mathrm{Ar}^{5+}$ and the spiral inflector area was pointed as a place of main beam losses [3]. To decrease the losses, a special magnetic channel was installed after the inflector.

The carried out optimisation, in couple with installation of two quadruple lenses before the first and the second bending magnets, did not give us essential improvement of the capture into acceleration efficiency in regimes with bunchers.

To study the place of losses more carefully, addition experimental investigations of the ion transportation efficiency from the second bending magnet to the cyclotron center have been done.

\section{INVESTIGATION OF THE BEAM TRANSPORTATION EFFICIENCY TO THE CYCLOTRON CENTER}

The beam transportation efficiency in the vertical part of injection line has been investigated by using the regular Faraday cup (FC) in vertical part of the line and special collectors, installed at the inflector entrance and exit. Also the positive (lower) electrode of the inflector was used for measurements of the current inside the inflector (Fig. 3).

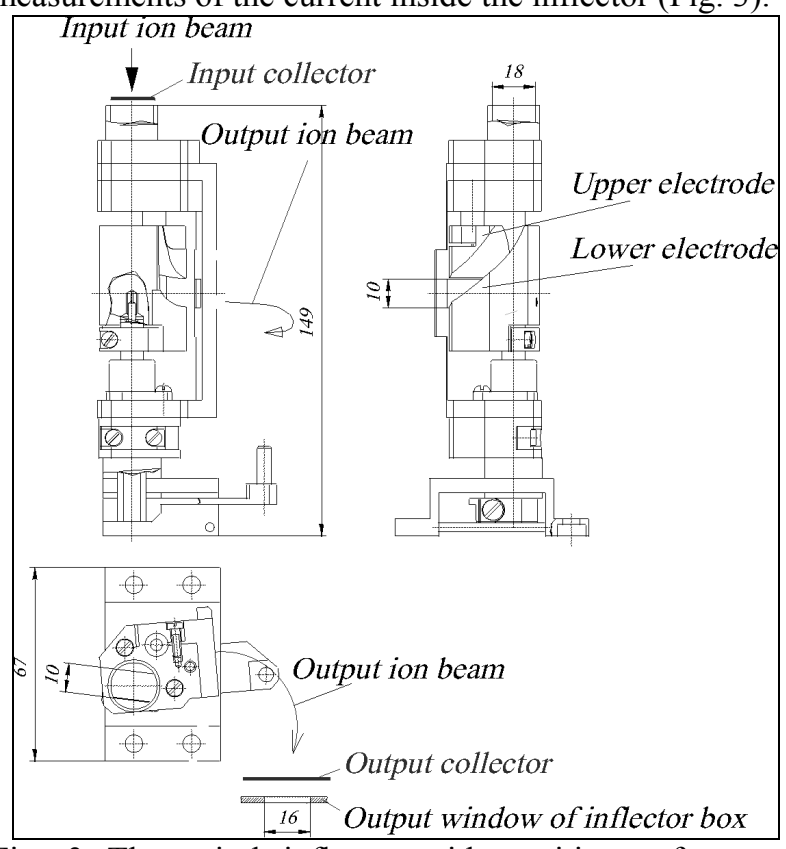

Fig. 3 The spiral inflector with positions of current collectors at its entrance and exit (input and output collectors).

At the first, the efficiency of current transportation of ${ }_{40} \mathrm{Ar}^{4+}$ ions from FC to the inflector input with the kinetic energy of $56.8 \mathrm{keV}$ was measured. The efficiency was calculated as a ratio between the current from lower inflector electrode (when the voltage at the electrodes was zero) and the current from FC in vertical part. The measured efficiency for the regime without bunchers was about $90 \%$ and for the regime with bunchers it was in the region of $74 \div 78 \%$ at the $\mathrm{FC}$ current values of $40 \div 50 \mu \mathrm{A}$.

Then, the collector (at first the big collector and then the small one) was installed at the inflector input. The collectors have been made form a thin cooper foil and were in a coin shape at the diameters of $30 \mathrm{~mm}$ (big) and $15 \mathrm{~mm}$ (small). The big collector diameter was more than the diameter of the hole in the magnetic plug (the plug with the diameter of $18 \mathrm{~mm}$ is situated above the inflector). The small collector diameter was close to the diameter of the inflector entrance. In the first experiment, the current value from the big collector was higher then the current from FC. We supposed that the current losses between the FC and inflector entrance were absent and the reason of the difference between the currents was the 
secondary electron emission from the collector. In the case, it can be the emission along the magnetic field. The difference between the currents was used to estimate of the emission coefficient. The calculated coefficient value was 1.6 , at accuracy of $14 \%$. The coefficient was taken into account for current measurements with the small collector. The results of the experiment are shown in the Fig. 4 We supposed that the efficiency for the close to zero currents is equal to $100 \%$. The linear fitting of the experimental results gives us that the maximal decreasing of measured efficiency takes place for the regime with two bunchers (from about $98 \%$ to $66 \%$ at the FC currents from 2 to $50 \mu \mathrm{A})$.

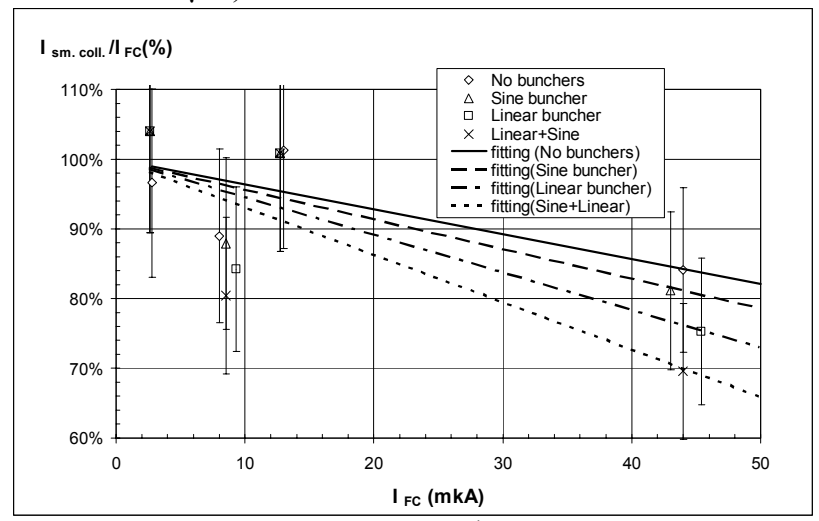

Fig. 4 The efficiency of the ${ }_{40} \mathrm{Ar}^{4+}$ ions transportation for the regimes without bunchers, with single sine buncher, with single linear buncher and with two bunchers.

Also, the currents at output collector have been measured at the working values of high voltage at the electrodes of $\pm 6.7 \mathrm{kV}$. The efficiency of ion current transportation throw the inflector was calculated as a ratio between the current from the output collector and the current from the lower inflector electrode. The measured efficiency value at the $\mathrm{FC}$ currents in the region of $12 \div 50 \mu \mathrm{A}$ was about $92 \%$ for three regimes: without bunchers, with the sine buncher and with the line one.

The influence of energy dispersion (the additional energy dispersion up to $3 \%$ appears after the bunchers) at the value of losses of ${ }_{40} \mathrm{Ar}^{4+}$ ions inside the inflector was studied by two ways. At the first, measurements of the efficiency of capture into acceleration had been carried out with constant voltages at the inflector electrodes and variation of the injection energy. Then, the injection energy was constant and the efficiency was measured with the symmetrical variation of voltage at the inflector electrodes. All the measurements were done without bunchers. In the first case, the results demonstrated that the ion injection energy deviation of $3 \%$ around $56.8 \mathrm{keV}$ leads to the maximal decreasing the efficiency by $5 \div 10 \%$. In the second case, the symmetrical deviation of the inflector voltages of $3 \%$ around $\pm 5.7 \mathrm{kV}$, corresponds to the maximal efficiency decreasing by $8 \div 10 \%$. The results of the second experiment are shown in the Fig. 5 as a percent ratio of the efficiency to maximal measured efficiency.

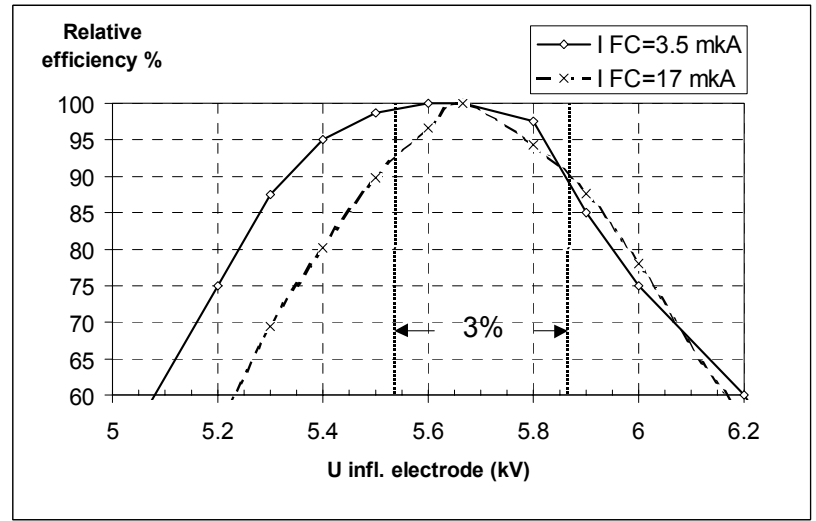

Fig. 5 The dependence of the relative efficiency of ${ }_{40} \mathrm{Ar}^{4+}$ acceleration from the voltage at the \pm inflector electrodes ( $U$ infl.electrode) for two values of ion current in the injection line (I FC). The injection energy was $56.8 \mathrm{keV}$.

\section{CONCLUSION}

The results show that the total losses at the inflector region can be more then $40 \%$ for the regimes with bunchers, at the FC current values above $40 \mu \mathrm{A}$. In the regime with bunchers, the place of main ion losses is the inflector entrance (more than 25\%). Increasing of the beam transverse emittance after the bunchers can be the reason of the losses. The effects of energy spread after the bunchers give not more than $10 \%$ of losses. To decrease the losses, the inflector transverse acceptance can be increased, but it is connected with mechanical difficulties because of the lack of free space in the region. Another way is reconstruction of the injection line to decrease its length from $9.28 \mathrm{~m}$ to $6.46 \mathrm{~m}$ with removing the $120^{\circ}$ bending magnet and optimisation of the injection line parameters. As a result of it, the beam transverse emittance before the inflector can be smaller and the ion losses can be essentially decreased [4].

\section{REFERENCES}

[1] Yu.Ts.Oganessian,G.G.Gulbekian, B.N.Gikal, M.ElShazly et al.,"Axial injection system for the U-400 cyclotron with the ECR-4M ion source", in JINR FLNR sci. rep.1995-1996, Heavy Ion Physics, pp. 270-276, Dubna 1997

[2] O. Borisiv, B. Gikal, G. Gulbekyan, I. Ivanenko, I. Kalagin, "Optimisation of the axial injection system for U-400 cyclotron (linear buncher)", in Proc. of EPAC2000, Vienna, Austria, pp. 1468-1470, 2000

[3] I. Ivanenko, G. Gulbekian, I. Kalagin, A. Morduev, "The compensation of beam vertical defocusing after the spiral inflector by using the passive magnetic channel", in Proc. of EPAC2000, Vienna, Austria, pp. 1504-1506, 2000

[4] V.S.Aleksandrov, G.G. Gulbekian, N.Yu.Kazarinov et al. "Numerical simulation of the $48 \mathrm{Ca} 5+$ ions transport along the U-400 cyclotron's injection line", in Cyclotrons2001 Conf., C01, Absrtacts, P4-02 